\title{
INVOLUTORIAL LINE TRANSFORMATIONS DETERMINED BY CREMONA PLANE INVOLUTIONS*
}

BY J. M. CLARKSON

1. Introduction. The author has discussed $\dagger$ an involutorial line transformation effected by considering a harmonic homology in each of two planes. If $A, B$ be the points in which an arbitrary line $(y)$ meets the planes $\alpha, \beta$, and if $A^{\prime}, B^{\prime}$ be their images by the homologies $I_{\alpha}, I_{\beta}$, respectively, then $(x) \equiv A^{\prime} B^{\prime}$ is the transform of $(y)$. It is the purpose of the present paper to consider the line transformations similarly determined by Cremona involutorial transformations in each of two planes. All combinations of the four fundamental types: Homology; Jonquières; Geiser; and Bertini will be considered. The orders of the transformations, the invariant loci, the singular elements and the transforms of certain elementary forms are discussed.

2. Homology-Jonquières. In the plane $\alpha$ consider a harmonic homology $I_{\alpha}$, center at $O_{1}$ and axis $\Delta_{\alpha}$. In the plane $\beta$ consider the perspective Jonquières involution $I_{\beta}$, of order $n$, with basis point $P_{1}$ of multiplicity $(n-1)$ and basis points $P_{2}, \cdots, P_{2 n-1}$ each simple, and with invariant curve $\Delta_{\beta}: P_{1}^{n-2} P_{2}^{1} \cdots P_{2 n-1}^{1}$ of order $n$ and genus $(n-2)$.

An arbitrary line $(y)$ meets $\alpha$ in a point $A$ whose coordinates are linear in the Plücker coordinates $y_{i}$ of $(y)$ and meets $\beta$ in a point $B$ whose coordinates are also linear in $y_{i}$. The image $A^{\prime}$ of $A$ by $I_{\alpha}$ has coordinates also linear in $y_{i}$ but the image $B^{\prime}$ of $B$ by $I_{\beta}$ has coordinates which are functions of degree $n$ in $y_{i}$. Hence $(x) \equiv A^{\prime} B^{\prime}$ has Plücker coordinates of degree $(n+1)$ in $y_{i}$. Thus, the transformation

$$
x_{i}=\phi_{i}(y)
$$

is of order $(n+1)$. The invariant lines of (1) form a congruence $(n, n)$ composed of the lines meeting $\Delta_{\alpha}, \Delta_{\beta}$; and in addition there is a cone of order $n$, vertex $O_{1}$, base curve $\Delta_{\beta}$.

\footnotetext{
* Presented to the Society, April 14, 1933.

$\dagger$ Some involutorial line transformations, this Bulletin, vol. 39 (1933), pp. 149-154.
} 
If $(y)$ meets $\beta$ in $P_{1}$ and $\alpha$ in $A$, then $(y) \sim$ a cone of order $(n-1)$, vertex $A^{\prime}$, base curve the curve of order $(n-1)$ into which $P_{1}$ is transformed by $I_{\beta}$. If $(y)$ meets $\beta$ in $P_{i}(i \neq 1)$ and $\alpha$ in $A$, then $(y) \sim$ the pencil whose vertex is $A^{\prime}$ and in the plane $A^{\prime} P_{1} P_{i}$.

The image by $I_{\alpha}$ of the line $c \equiv \alpha \beta$ is a line $c_{\alpha}$ and by $I_{\beta}$ is a curve $c_{\beta}$ of order $n$. The points $C_{\alpha}$ of $c_{\alpha}$ and $C_{\beta}$ of $c_{\beta}$ are projective with the points $C$ of $c$. Hence the line joining any two corresponding points $C_{\alpha}, C_{\beta}$ is transformed by (1) into the bundle whose vertex is $C$. These lines $C_{\alpha} C_{\beta}$ form a regulus $\{c\}^{n+1}$ of order $(n+1)$, every generator of which is singular.

A line $t_{\alpha}$ in $\alpha$ meets $\beta$ in a point $C$ whose image by $I_{\beta}$ is $C_{\beta}$. The image of $t_{\alpha}$ by $I_{\alpha}$ is a line $t_{\alpha}^{\prime}$. Hence $(y) \equiv t_{\alpha} \sim$ a pencil, vertex $C_{\beta}$, plane $C_{\beta} t_{\alpha}^{\prime}$. If $t_{\alpha}$ pass through $O_{1}$, then $t_{\alpha}^{\prime} \equiv t_{\alpha}$ and the plane of the pencil is $C_{\beta} t_{\alpha}$. If $t_{\alpha}$ be the axis $\Delta_{\alpha}$, then $t_{\alpha}^{\prime} \equiv \Delta_{\alpha}$ and the plane of the pencil is $C_{\beta} \Delta_{\alpha}$.

A line $t_{\beta}$ in $\beta$ meets $\alpha$ in a point $C$ whose image by $I_{\alpha}$ is $C_{\alpha}$. The image of $t_{\beta}$ by $I_{\beta}$ is a curve $\rho_{\beta}$ of order $n$. Hence $(y) \equiv t_{\beta} \sim$ a cone of order $n$, vertex $C_{\alpha}$, base curve $\rho_{\beta}$. If $t_{\beta}$ pass through $P_{1}$, then $\rho_{\beta}$ is a line, and indeed the line $t_{\beta}$. Hence the conjugate is no longer a cone but a pencil, vertex $C_{\alpha}$, plane $C_{\alpha} t_{\beta}$. If $t_{\beta}$ pass through $P_{i}(i \neq 1)$, then $\rho_{\beta}$ is of order $(n-1)$ and the conjugate cone is of order $(n-1)$. If $t_{\beta}$ be the line $P_{1} P_{i}$, then $(y) \equiv t_{\beta} \sim C_{\alpha} P_{i}$.

As $t_{\alpha}$ describes the pencil $(C, \alpha)$ each conjugate pencil has its vertex at $C_{\beta}$ and its plane passes through $C_{\alpha}$. As $C$ describes $c$, $C_{\beta}$ describes $c_{\beta}$, and hence the plane field $(\alpha) \sim$ the special complex of order $n$ with $c_{\beta}$ as directrix curve.

As $t_{\beta}$ describes the pencil $(C, \beta)$, then each conjugate cone has its vertex at $C_{\alpha}$ and has $C_{\alpha} C_{\beta}$ as a generator. As $C$ describes $c, C_{\alpha}$ describes $c_{\alpha}$, and hence the plane field $(\beta) \sim$ the special linear complex $\left|c_{\alpha}\right|$.

Each line $t_{\alpha}$ of a pencil $(T, \alpha)$ is transformed by (1) into a pencil whose vertex is on $c_{\beta}$ and whose plane passes through $t_{\alpha}^{\prime}$ which belongs to the pencil $\left(T^{\prime}, \alpha\right), T^{\prime}$ being the image by $I_{\alpha}$ of $T$. These $\infty^{1}$ pencils form a congruence $(n+1, n)$. If a line $(x)$ meet $c_{\beta}$ in a point $C_{\beta}$ and also meet the $t_{\alpha}^{\prime}$ which corresponds to $C_{\beta}$, then $(x)$ belongs to the conjugate congruence. Through an arbitrary point of space a line $(x)$ belonging to the conjugate congruence has coordinates which are of degree $(n+1)$ in the parameter $\lambda$ of a line of $(T, \alpha)$. Hence the order of the con- 
gruence is $(n+1)$. An arbitrary plane meets $c_{\beta}$ in $n$ points and meets each corresponding $t_{\alpha}^{\prime}$ in one point. Hence the class is $n$.

Likewise a pencil $(T, \beta) \sim$ a congruence $(n+1, n)$. However, if $T$ lie at $P_{1}$, the order and class are both reduced so that the congruence is $(2,1)$. If $T$ lie at $P_{i}(i \neq 1)$, then the congruence is $(n, n-1)$.

An arbitrary pencil $(T, \tau) \sim$ a regulus $R$ of order $(n+1)$, the generators of which are the joins of corresponding points on the straight line image by $I_{\alpha}$ of $\tau \alpha$ and the curve of order $n$, image by $I_{\beta}$ of $\tau \beta$. If $\tau$ pass through $P_{1}$, then $R$ is of order 2 ; through $P_{i}(i \neq 1)$, of order $n$; through $P_{i} P_{j}(i, j \neq 1)$, of order $(n-1)$; through $P_{1} P_{i}$, the conjugate is a pencil with vertex $P_{i}$.

An arbitrary plane field of lines $(\tau) \sim$ a congruence $(n, n)$ composed of lines meeting a line and a plane curve of order $n$ not meeting the line. If $\tau$ pass through $P_{1}$, the congruence is $(1,1)$; through $P_{i}(i \neq 1),(n-1, n-1)$; through $P_{i} P_{j}(i, j \neq 1)$, $(n-2, n-2)$; through $P_{1} P_{i}$, the conjugate is no longer a congruence but a pencil, vertex $P_{i}$.

An arbitrary bundle $(T) \sim$ a congruence $(3 n, n)$. From an arbitrary point of space, the points of the planes $\alpha, \beta$ form two projective fields. There are three coincidences in a section of such a projection. Hence the parameters $\lambda, \mu$ of a line of $(T)$ appear to degree $3 n$ in defining a line $(x)$ of the conjugate congruence through an arbitrary point of space. In an arbitrary plane of space lie $n$ lines of the congruence.

A bilinear congruence $\left[\left|d_{1}\right|,\left|d_{2}\right|\right] \sim$ a congruence $(4 n, 2 n)$. The transformation (1) is involutorial. Hence the conjugate congruence of the $(1,1)$ will be of order equal to the number of lines common to the $(1,1)$ and the conjugate of an arbitrary bundle, which is $4 n$. Likewise, the class will be the number of lines common to the $(1,1)$ and the conjugate of an arbitrary plane field.

A linear complex is transformed by (1) into a complex of order $(n+1)$ since this is the order of the transformation.

3. Homology-Geiser. Consider $I_{\alpha}$ in $\alpha$ as before, and in $\beta$, $I_{\beta}: P_{1}^{3} \cdots P_{7^{3}}$, with invariant curve $\Delta_{\beta}$ of order 6 having double-points at $P_{1}, \cdots, P_{7}$. The order of the transformation is 9 ; the invariant lines form a congruence $(6,6)$ and a cone of order 6 , vertex $O_{1}$, base curve $\Delta_{\beta}$; the singular elements are, as 
before, the lines of the bundles whose vertices are $F$-points of $I_{\beta}$, a regulus $\{c\}^{9}$ of order 9 and the plane fields $(\alpha),(\beta)$. To any line of any bundle corresponds a cone of order 3 ; to any generator of $\{c\}{ }^{9}$, a bundle, vertex on $c$; to $(\alpha)$ a special complex of order 8 , and to $(\beta)$ a special linear complex. If $t_{\beta}$ pass through one or two points $P_{i}$, its conjugate cone is of order 5 or 2 . If the vertex $T$ of a pencil $(T, \beta)$ be at $P_{i}$, the conjugate congruence of the pencil is $(6,5)$; otherwise $(T, \alpha)$ or $(T, \beta) \sim$ a congruence $(9,8)$.

If the plane $\tau$ of an arbitrary pencil $(T, \tau)$ pass through one or two points $P_{i}$, the conjugate regulus is of order 6 or 3 . Otherwise $(T, \tau) \sim$ a regulus of order 9.

If the plane $\tau$ pass through $j$ points $P_{i}(j=0,1,2),(\tau) \sim$ a congruence $(8-3 j, 8-3 j)$.

An arbitrary bundle $(T) \sim$ a congruence $(24,8)$.

A congruence $(1,1) \sim$ a congruence $(32,16)$, and a linear complex $\sim$ a complex of order 9 .

4. Homology-Bertini. Consider $I_{\alpha}$ as before and $I_{\beta}: P_{1}{ }^{6} \cdots P_{8}{ }^{6}$, $\Delta_{\beta}$ being of order 9 with triple-points at $P_{i}$. The order of the transformation is 18; the invariant lines form a congruence $(9,9)$ and a cone of order 9 ; the singular elements are the lines of the bundles $\left(P_{i}\right)$, a regulus $\{c\}^{18}$ of order 18 , and the plane fields $(\alpha),(\beta)$. Any line of any $\left(P_{i}\right) \sim$ a cone of order 6 , each generator of $\{c\}^{18} \sim$ a bundle with vertex on $c,(\alpha) \sim$ a special complex of order 17 , and $(\beta) \sim$ a special linear complex. The conjugate cone of a line $t_{\beta}$ has its order $17-6 j$, where $j$ is the number of points $P_{i}$ on $t_{\beta}$. The conjugate congruence of a pencil $(T, \alpha)$ or $(T, \beta)$ is $(18,17)$ unless $T$ lie at some $P_{i}$, when the congruence is $(12,11)$.

If $\tau$ pass through $j$ points $P_{i}(j=0,1,2)$, then the pencil $(T, \tau) \sim$ a regulus of order $18-6 j$ and the plane field $(\tau) \sim$ a congruence $(17-6 j, 17-6 j)$.

A bundle $(T) \sim$ a congruence $(51,17)$.

A congruence $(1,1) \sim$ a congruence $(68,34)$, and the transform of a linear complex is a complex of order 18 .

5. Jonquières-Jonquières. When we consider two perspective Jonquières involutions, $I_{\alpha}$ of order $m$, center $O_{1}$, and $I_{\beta}$ of order $n$, center $P_{1}$, the order of the transformation is $(m+n)$. The invariant lines form a congruence $(m n, m n)$ since now $\Delta_{\alpha}$ and $\Delta_{\beta}$ 
are of orders $m, n$, respectively, and each passes simply through the simple $F$-points in its respective plane and multiply through the center. In addition to the singular regulus $\{c\}^{m+n}$ of order $(m+n)$ whose generators are transformed into bundles with vertices on $c$, and the singular plane fields $(\alpha),(\beta)$ whose conjugates are special complexes of orders $n, m$, respectively, and the singular bundles whose vertices are at $F$-points and each of whose lines is transformed into a cone whose order is the multiplicity of the $F$-point in $I_{\alpha}$ or $I_{\beta}$, there are $(2 m-1)(2 n-1)$ singular lines $O_{i} P_{j}$ whose conjugates are congruences. If $i, j \neq 1$, each congruence is $(1,1)$; if $i=1, j \neq 1$, each congruence is $(m, m)$; if $i \neq 1, j=1$, each is $(n, n)$; if $i=j=1$, the conjugate congruence is $([m-1][n-1],[m-1][n-1])$. A line $(y) \equiv t_{\alpha} \sim$ a cone of order $(n-j)$, where $j$ is the sum of the multiplicities of $F$-points $O_{i}$ on $t_{\alpha}$, and $(y) \equiv t_{\beta} \sim$ a cone of order $(m-i)$, where $i$ is the sum of the multiplicities of $F$-points $P_{l}$ on $t_{\beta}$. A pencil $(T, \beta) \sim$ a congruence $(m+n-i, m[n-i])$ where $i$ is the multiplicity of $T$ as an $F$-point of $I_{\beta}$ and $(T, \alpha) \sim$ a congruence $(m-j+n,[m-j] n)$, where $j$ is the multiplicity of $T$ as an $F$-point of $I_{\alpha}$.

An arbitrary pencil $(T, \tau) \sim$ a regulus $R$ of $\operatorname{order}(m-i+n-j)$, where $i$ is the sum of the multiplicities of $F$-points $O_{k}$ and $j$ the sum of the multiplicities of $F$-points $P_{l}$ lying in $\tau$. If $i=m$ or $j=n$, then $R$ is a cone. Both $i=m$ and $j=n$ will not occur if $I_{\alpha}, I_{\beta}$ be taken arbitrarily.

A plane field $(\tau) \sim$ a congruence whose order and class are both $(m-i)(n-j)$, where $i, j$ are as defined immediately above. In the event $m-i=0$ or $n-j=0$, the conjugate of $(\tau)$ is a cone whose order is the factor which does not vanish.

An arbitrary bundle $(T) \sim$ a congruence $(3 m n, m n)$.

A congruence $(1,1) \sim$ a congruence $(4 m n, 2 m n)$, and a linear complex has for conjugate a complex of order $(m+n)$.

6. Jonquières-Geiser. Take $I_{\alpha}$ as in $\$ 5$ and $I_{\beta}$ as in $\S 3$. The transformation is of order $(m+8)$; the invariant lines form a congruence $(6 m, 6 m)$; the singular elements are the bundles $\left(O_{i}\right),\left(P_{j}\right)$, the $7(2 m-1)$ lines $O_{i} P_{j}$, the plane fields $(\alpha),(\beta)$, and the singular regulus $\{c\}^{m+8}$ defined as in each previous case. Each line of $\left(O_{i}\right) \sim$ a cone of order $(m-k)$, where $k$ is the multiplicity of $O_{i}$ in $I_{\alpha}$, and each line of $\left(P_{j}\right) \sim$ a cone of order 3; 
$(y) \equiv O_{i} P_{j} \sim$ a congruence whose order and class are both $5(m-k)$, where $k$ is as defined just above; $(\alpha) \sim$ a special complex of order 8 and $(\beta) \sim$ a special complex of order $m$; each generator of $\{c\}^{m+8} \sim$ a bundle whose vertex is on $c$. Finally $(y) \equiv t_{\alpha} \sim$ a cone of order $(m-i)$, where $i$ is the sum of the multiplicities of $F$-points on $t_{\alpha}$, and $(y) \equiv t_{\beta} \sim$ a cone of order $(8-j)$, where $j$ is the sum of the multiplicities of $F$-points on $t_{\beta}$.

A pencil $(T, \alpha) \sim$ a congruence $(m-i+8,[m-i] 8)$, where $i$ is the multiplicity of $T$ as an $F$-point of $I_{\alpha}$, and a pencil $(T, \beta) \sim$ a congruence $(m+8-j, m[8-j])$, where $j$ is the multiplicity of $T$ as an $F$-point of $I_{\beta}$.

An arbitrary pencil $(T, \tau) \sim$ a regulus $R$ whose order is $(m-i+8-j)$, where $i$ is the sum of multiplicities of $F$-points $O_{k}$ and $j$ the sum of multiplicities of $F$-points $P_{l}$ lying in $\tau$. If $I_{\alpha}, I_{\beta}$ be taken arbitrarily, no more than 3 points $O_{k}, P_{l}$ lie on $\tau$. If $i=m, R$ is a cone of order $(8-j)$.

A plane field $(\tau) \sim$ a congruence whose order and class are both $(m-i)(8-j)$, where $i, j$ are as defined in the preceding paragraph. If $i=m$, the conjugate of $(\tau)$ is a cone of order $(8-j)$.

A bundle $(T) \sim$ a congruence $(24 m, 8 m)$.

A congruence $(1,1) \sim$ a congruence $(32 m, 16 m)$ and a linear complex is transformed into complex of order $(m+8)$.

7. Jonquières-Bertini. Take $I_{\alpha}$ as in $\S 5$ and $I_{\beta}$ as in $\S 4$. The transformation is of order $(m+17)$, and all of the results follow by replacing 8 by 17 in the preceding section, except that a bundle $(T) \sim$ a congruence $(51 \mathrm{~m}, 17 \mathrm{~m})$ and a congruence $(1,1) \sim$ a congruence $(68 \mathrm{~m}, 34 \mathrm{~m})$.

8. Geiser-Geiser. Given $I_{\alpha}: O_{1}^{3} \cdots O_{7}^{3}$ and $I_{\beta}$ as in $\$ 3$. The transformation is of order 16; the invariant lines form a congruence $(36,36)$; the singular elements are $\left(O_{i}\right),\left(P_{j}\right), O_{i} P_{j}$, $\{c\}{ }^{16},(\alpha),(\beta)$, and their conjugates are easily found by the methods outlined above. The conjugates of the elementary forms $(T, \tau),(\tau),(T),(1,1)$ and linear complex are also readily obtained.

9. Geiser-Bertini. Consider $I_{\alpha}$ as in $\S 8$ and $I_{\beta}$ as in $\S 4$. The transformation is of order 25; the invariant lines form a congruence $(54,54)$; the singular elements are $\left(O_{i}\right),\left(P_{j}\right), O_{i} P_{j}$, $\{c\}{ }^{25},(\alpha),(\beta)$, and their conjugates, and those of the elemen- 
tary forms follow immediately from the method we have used throughout this paper.

10. Bertini-Bertini. Given $I_{\alpha}: O_{1}{ }^{6} \cdots O_{8}{ }^{6}$ and $I_{\beta}$ as in $\S 4$. The order of the transformation is 34 , and by repetition of what has been done above we can discuss this transformation completely.

If fundamental elements of one or both Cremona plane involutions lie on the line $c \equiv \alpha \beta$ the preceding results must be modified. The details are not difficult in each particular case, but the large number of possible forms cannot be considered here.

Cornell University

\section{A SEPARATION THEOREM*}

BY W. A. WILSON

Various writers on topology have had occasion in the course of their work to prove lemmas of the following general nature. Given sets $A$ and $B$ lying in a connected space $Z$, it is possible to express $Z$ as the union of two continua $M$ and $N$ such that $N \cdot(A-A \cdot B)=M \cdot(B-A \cdot B)=0$, provided that $A, B$, and $Z$ satisfy the proper conditions. The last of these to come to the writer's attention are two theorems by Vietoris and one by the author of this note. $\dagger$ Such theorems are of course generalizations of Tietze's separation axioms $\ddagger$ and it might prove profitable to work out systematically the possibilities along this line.

In some of the generalizations mentioned it is shown that, if $Z$ is locally connected, a decomposition $Z=M+N$, where $M$ and $N$ are also locally connected, is possible, but, as far as the writer knows, the following theorem, which shows a certain kind of local connectivity for $M \cdot N$ as well as for $M$ and $N$, is new.

Theorem. Let $A$ and $B$ be sub-continua of the locally connected compact metric space $Z$ and let $A \cdot B$ be totally disconnected or void.

* Presented to the Society, February 25, 1933.

$\dagger$ L. Vietoris, Über den höheren Zusammenhang, Fundamenta Mathematicae, vol. 19, pp. 271-272; W. A. Wilson, On unicoherency about a simple closed curve, American Journal of Mathematics, vol. 55, p. 141.

¥ See F. Hausdorff, Mengenlehre, p. 229. 